\title{
BMJ Open Identifying risk factors for neurological complications and monitoring long-term neurological sequelae: protocol for the Guangzhou prospective cohort study on hand-foot-and-mouth disease
}

\author{
Kuanrong Li, ${ }^{1}$ Xufang $\mathrm{Li}^{2}{ }^{2}$ Wenyue $\mathrm{Si},{ }^{1}$ Huiying Liang, ${ }^{1}$ Hui-Min Xia, ${ }^{3} \mathrm{Yi} \mathrm{Xu}^{2}$
}

To cite: Li K, Li X, Si W, et al. Identifying risk factors for neurological complications and monitoring long-term neurological sequelae: protocol for the Guangzhou prospective cohort study on hand-footand-mouth disease. BMJ Open 2019;9:e027224. doi:10.1136/ bmjopen-2018-027224

- Prepublication history and additional material for this paper are available online. To view these files, please visit the journal online (http://dx.doi org/10.1136/bmjopen-2018027224).

Received 15 0ctober 2018 Revised 11 January 2019 Accepted 28 January 2019

Check for updates

(c) Author(s) (or their employer(s)) 2019. Re-use permitted under CC BY-NC. No commercial re-use. See rights and permissions. Published by BMJ.

${ }^{1}$ Institute of Paediatrics, Guangzhou Women and Children's Medical Center, Guangzhou, China

${ }^{2}$ Department of Infectious Diseases, Guangzhou Women and Children's Medical Center, Guangzhou, China

${ }^{3}$ Division of Birth Cohort Study, Guangzhou Women and Children's Medical Center, Guangzhou Medical University, Guangzhou, China

Correspondence to Dr Yi Xu; xuyi70@163.com

\section{ABSTRACT}

Introduction Hand-foot-and-mouth disease (HFMD) is a paediatric infectious disease that is particularly prevalent in China. Severe HFMDs characterised by neurological involvement are fatal and survivors who have apparently fully recovered might still be afflicted later in life with neurocognitive impairments. Only when a well-designed, prospective cohort study is in place can we develop clinical tools for early warning of neurological involvement and can we obtain epidemiological evidence regarding the lingering effects of the sequelea.

Methods and analysis A prospective, hospital-based cohort study is underway in Guangzhou, China. Clinical data and biosamples from hospitalised children $(<14$ years of age) with an admission diagnosis of HFMD will be collected to determine risk factors for subsequent neurological involvement. Clinical tools for early detection of severe HFMDs will be developed by integrating clinical and biological information. Questionnaire surveys and neurocognitive assessments will be conducted at discharge and each year in the first 2 years of follow-up and every 2 years afterwards until study participants turn 16 years of age or show no evidence of neurocognitive deficits. The association between childhood enterovirus infection and neurocognitive impairment later in life will be examined.

Ethics and dissemination A written informed consent from parents/guardians is a prerequisite for study entry. The protocol of this study has been approved by the hospital's ethics committee. Data usage follows the rules of the hospital's data oversight committee. Findings of this study will be disseminated through publications in international peer-reviewed journals and will be presented in academic conferences.

Trial registration number ChiCTR-E0C-17013293; Preresults.

\section{INTRODUCTION}

Hand-foot-and-mouth disease (HFMD) is a common paediatric infectious disease characterised by rashes/blisters mainly in hands and feet and ulcers in oral cavity. Enterovirus A 71 (EV-A71) and coxsackievirus A 16
Strengths and limitations of this study

- The long-term neurocognitive impairment subsequent to severe hand-foot-and-mouth disease has been examined by very few prospective studies. This study is a worthy addition with its much larger cohort and more thorough, consecutive assessments of neurocognitive abilities.

- This study collects detailed clinical and biological data to identify patients with increased risk of developing neurological complications.

- The potential limitations of this study include selection bias during recruitment and follow-up (as a result of loss to follow-up) and the problem of missing data.

(CV-A16) are the two major causative agents of the disease, while another serotype CV-A6 has emerged as a predominant pathogen behind some of the recent outbreaks in Asia and Europe ${ }^{1-7}$ HFMD is particularly prevalent in the west Pacific region, where China is the country that is affected the most, with 13 million HFMDs having been reported during the period from 2008 to $2015 .{ }^{8}$ The HFMD epidemic distributes unevenly across China-the highest incidence rate $(>30$ per 10000 person-years, equivalent to four times the national level) occurs in Guangdong Province, partly due to its subtropical climate. ${ }^{910}$

HFMD is mostly a self-limiting illness. However, nearly $1 \%$ of HFMD patients develop neurological and/or cardiopulmonary complications such as encephalitis, aseptic meningitis and cardiorespiratory failure. ${ }^{11}$ It is crucial yet challenging for clinicians to spot HFMD patients who are at high risk of central nervous system (CNS) involvement among a large number of mild cases. Some clinical or laboratory features such as persistent high fever, lethargy, elevated 
neutrophil count, cerebrospinal fluid (CSF) pleocytosis and EV-A71 infection might be potential warning signs for CNS involvement. ${ }^{12-14}$ Based on these factors, several risk scores and algorithms have been developed for early warning purpose ${ }^{15-17}$ However, all these risk factors were identified retrospectively in small studies. Thus their usefulness and accordingly the value of the existing risk scores/algorithms are questionable.

In China, approximately $90 \%$ of the incident HFMDs occur among children under the age of 5 years. ${ }^{12}$ The crucial period for humans' cognitive development is the first several years of life; therefore the deleterious neurocognitive impact of CNS complications (eg, encephalitis) subsequent to HFMD is likely to be substantial and enduring. Few studies, with a limited number of children $(\mathrm{n}<150)$ and short follow-up period (median $<3$ years), have observed neurocognitive developmental deficits following severe EV-A17 infection. ${ }^{18} 19$ There is thus a need for more conclusive findings from prospective studies where the patient cohort is large, the neurocognitive assessment is comprehensive, and the follow-up period is long.

In this protocol, we describe in detail the rationale and design of an ongoing hospital-based cohort study-the Guangzhou Prospective Cohort Study on Hand-Foot-andMouth Disease (GPCS-HFMD) - in Guangdong Province, China. This study is being conducted in the Guangzhou Women and Children's Medical Centre (GWCMC), which is the largest tertiary referral paediatric hospital in Southern China. The objective of the GPCS-HFMD study is three-fold: (1) to develop early warning tools for CNS involvement using early biomarkers and clinical manifestations; (2) to assess the long-term neurocognitive impairment after severe HFMD; and (3) to build an HFMD biobank for future research activities.

\section{METHODS AND ANALYSIS}

\section{Study design and participant recruitment}

The recruitment of study participants has started since the first of December 2017. Consecutive inpatients who are admitted to the infectious disease wards with an HFMD diagnosis after that date are considered for their eligibility to enter the cohort. HFMD diagnosis and decision for hospitalisation are made following the National Health Commission's Guideline on Hand-Foot-andMouth Disease Diagnosis and Treatment (2018 edition). ${ }^{20}$ Inpatients are excluded if they have one or more of the following criteria: (1) $\geq 14$ years of age at admission; (2) complicated with any pathogenically confirmed viral and/ or bacterial infections at admission that might confound the CNS and pulmonary complications associated with HFMD (eg, herpes simplex virus and adenovirus); (3) have physical/mental retardation at admission; (4) have any underlying neurological, systematic, hereditary or metabolic diseases; and (5) lack of written informed parental/guardian consent. According to the study plan,

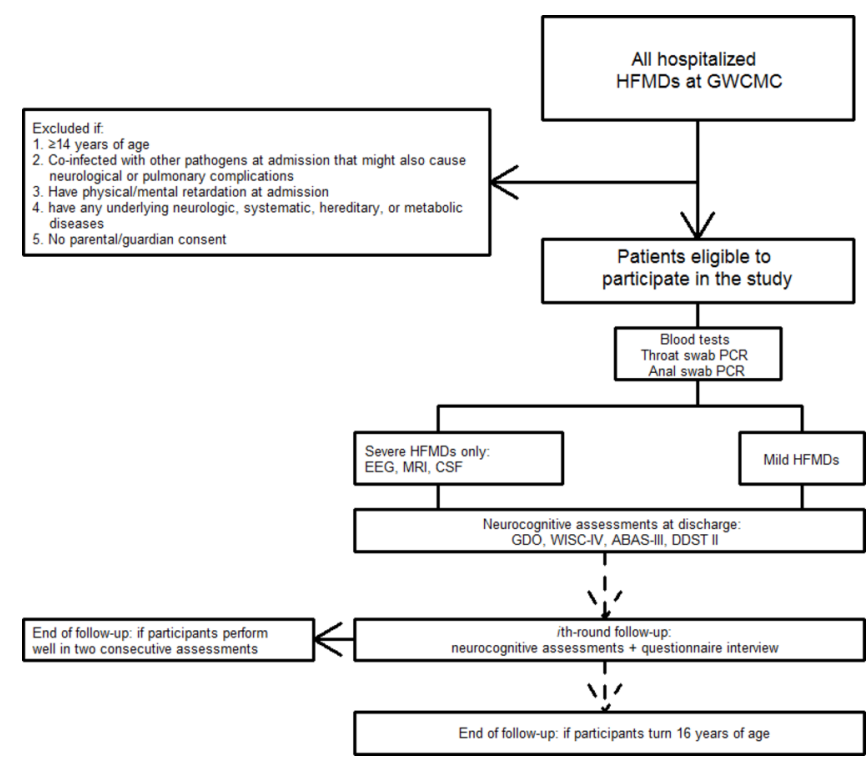

Figure 1 Cohort diagram of the Guangzhou prospective study on hand-foot-and-mouth disease. ABAS-III,

Adaptive Behavior Assessment System the Third Edition; CSF, cerebrospinal fluid; DDST II, Denver Developmental Screening Test; EEG, electroencephalography; GDO, Gesell Developmental Observation; GWCMC, Guangzhou Women and Children's Medical Centre; HFMD, hand-foot-and-mouth disease; WISC-IV: Wechsler Intelligence Scale the Fourth Edition.

the recruitment will continue until the year 2023. figure 1 provides an overall view of the study design.

In the past 5 years, we admitted averagely 1200 patients with HFMD each year; among them around $10 \%$ developed CNS complications (online supplementary appendix figure). We thus expect that after exclusions, our cohort will recruit more than 4000 patients including about 400 cases with CNS involvement by the end of recruitment. A prospective cohort of this size has a statistical power of $80 \%$ to detect a moderate relative risk of 1.5 for a less common predictor (prevalence of the Predictor $=10 \%$ ), given a probability of 0.05 for type I error, an overall incidence of $10 \%$ for the event of interest, and a rate of $10 \%$ for loss to follow-up.

\section{Data collection}

Baseline clinical and laboratory data

Administrative, demographic and routine clinical data are collected during the initial outpatient department visit. Biosamples including blood, throat swab and anal swab are collected and assayed for all study participants at both admission and discharge. For each patient who has been enrolled in the cohort, a virological diagnosis will be made using throat/anal swab PCR technique. Extra swabs (treated with preservatives in $15 \mathrm{~mL}$ vials) and aliquots of blood samples $(4 \mathrm{~mL}$, whole venous blood, anticoagulated with EDTA) will be stored in a $-70^{\circ} \mathrm{C}$ freezer. For severe HFMDs, CSF test, electroencephalography and MRI are performed additionally to confirm brain involvement. An aliquot of CSF $\left(2 \mathrm{~mL}\right.$ ) will be stored in a $-70^{\circ} \mathrm{C}$ freezer for 
future analysis. Colour Doppler ultrasound and pulmonary function tests are also provided but only if they are clinically necessary. All study participants are also given a thorough neurocognitive assessment before discharge (details about the assessment are described below).

Definition of central nervous system involvement and severe handfoot-and-mouth disease

CNS involvement is defined using the established criteria, that is, the presence of any of the following features: aseptic meningitis, encephalitis, encephalomyelitis, acute flaccid paralysis or autonomic nervous system dysregulation, whichever comes first. ${ }^{11}$ Severe HFMD is defined as the presence of either CNS complication or cardiopulmonary complication (pulmonary oedema, pulmonary haemorrhage or cardiorespiratory failure), or both. ${ }^{11}$

\section{Follow-up and neurocognitive assessments}

During the follow-up, research nurses telephone parents/ guardians of the participating children every 6 months to collect the information about the occurrence of any disease in the last 6 months. This information is verified if the disease was diagnosed at the GWCMC using the electronic medical record system. Parents/guardians are also advised in particular to notify the research nurses of any HFMD-like symptoms so that a hospital visit for medical diagnosis can be scheduled. In an effort to maintain a high follow-up rate, those who do not respond to the first phone call will be contacted again within 2 days. Newsletters will be sent to parents/guardians regularly via social media apps to keep them informed of the progress and achievements of the study.

A questionnaire interview regarding the general health condition and a comprehensive neurocognitive assessment will be conducted each year in the first 2 years of follow-up and every 2 years afterwards. Neurocognitive development will be assessed by independent professional neuropsychologists using the Gesell Developmental Observation for children under the age of 3 years and the Wechsler Intelligence Scale the Fourth Edition for those over this age. ${ }^{21} 22$ The adaptive skills of children over the age of 6 months will be tested using the Adaptive Behaviour Assessment System the Third Edition. ${ }^{23}$ For children under the age of 6 years, their gross and fine motor skills will be assessed with the Denver Developmental Screening Test. ${ }^{24}$

\section{Patient and public involvement}

Neither patients nor their parents/guardians, nor the public were involved in the study design. Any published results will be simplified using plain language and then sent to the parents/guardians.

\section{Statistical analysis}

Characteristics of the study participants will be summarised using descriptive statistical methods. Bioinformatic technologies will be used to analyse the 'omics' data derived from biosamples for identification of promising precursory biomarkers. Statistical regression models (eg, Cox proportional hazards model) and machine learning approaches will be applied to develop clinical tools that can be used for early detection of patients at high risk of neurological complications. Risk prediction using case-cohort design will be considered given its advantage that expensive examinations/tests (eg, genotyping) can be restricted to a much smaller subcohort. In this case a weighted Cox proportional hazards model will be fitted. The discriminative performance of the clinical tools will be evaluated internally and possibly externally. Data on neurocognitive assessments will be compared between mild and severe HFMDs using different statistical methods depending on the distribution of the data. All the reported $p$ values will be two-sided with 0.05 as the threshold for statistical significance.

\section{DISCUSSION}

Outbreak of HFMD is common in China and over a million young children are affected each year. Severe HFMD is of great concern to the healthcare authority given the avoidable loss of hundreds of lives it causes annually. ${ }^{11}$ According to the latest Chinese national guideline on HFMD diagnosis, the presence of the following physical or laboratory signs indicate the need for intensive monitoring: persistent fever $\left(>39^{\circ} \mathrm{C}\right)$, lethargy, myoclonic jerks, tachycardia, tachypnoea, hyperglycaemia, increased neutrophil count and increased blood lactate level. ${ }^{20}$ However, no prognostic tool is available for early detection of severe HFMDs before the listed clinical manifestations become evident.

Several risk prediction algorithms based on clinical symptoms and results of laboratory tests for developing severe HFMD have been built, but whether they would be adopted by clinicians remains in doubt because of the uncertainty about their clinical advantages. Moreover, it is unclear to what degree those predictors precede the onset of neurological complications. This is a particularly important concern considering the fact that most HFMDs develop neurological complications only 1 day after admission. ${ }^{15}$ A number of single nucleotide polymorphisms have been identified as risk factors for severe EV-A71 infection, ${ }^{25-27}$ but polymorphism testing may take several days. With the detailed clinical and biological information it collects, the GPCS-HFMD study is expected to discover some meaningful and applicable early biomarkers that can contribute to early prediction and prevention of disease deterioration.

For survivors of severe HFMD, childhood infection of EV-A71 might lead to lifelong neurological and cognitive impairment. Previous studies have shown a substantial burden of neurological sequelae following severe HFMD ${ }^{28}$ Delayed neurodevelopment and reduced cognitive functioning after encephalitis following EV-A71 infection have been observed in two small-sized cohorts, ${ }^{18} 19$ but findings from large-scale prospective cohorts are still in absence. One case-control study found an increased risk of attention deficit hyperactivity disorder associated 
with enterovirus encephalitis. ${ }^{29}{ }^{30}$ Autism spectrum disorder cases secondary to enterovirus encephalitis have also been reported sporadically. ${ }^{31} 32$ However, only largescale prospective patient cohort studies are likely to establish convincing epidemiological associations between childhood EV-A71 infection and subsequent neurological/cognitive disorders given the low incidence rates of these outcomes and the involvement of numerous confounding factors. To the best of our knowledge, the GPCS-HFMD study has been the first prospective cohort study so far that is designed to evaluate the long-term neurocognitive effects following childhood EV-A71 infection, and thus this study will yield important insights into the overall burden of the disease. Compared with EV-A71, CV-A6 and CV-A16 rarely cause CNS complications. 33 However, more studies are needed to confirm this and to investigate the underlying mechanisms.

Selection bias is a major limitation of this study owing to the study's hospital-based setting and prospective nature. As a tertiary referral hospital in the region, our institute is likely to receive more HFMD patients with severe symptoms; on the other hand, when overwhelmed by a large number of patients seeking medical care during HFMD outbreaks, doctors have to be selective about who should be admitted. Both circumstances will introduce selection bias at the recruitment stage. Selection bias would also arise during follow-up as it might be difficult for very healthy and/or very sick children to maintain a long-term participation in our study. It should be noted that we do not place a restriction on the time from disease onset to diagnosis/admission, as doing so will introduce further selection bias. Incompleteness of data is another possible limitation, because some invasive or expensive examinations (eg, lumbar puncture and MRI) are conditioned upon parental consent.

In summary, severe HFMD is a fatal paediatric disease that necessitates early detection for better prognosis as well as a continuous monitoring of long-term neurocognitive consequences. The upcoming findings of the GPCS-HFMD study will provide primary knowledge on better approaches to early detection and treatment of severe HFMD and will also lead to development of timely posthospital intervention strategies against its neurocognitive sequelae.

\section{ETHICS AND DISSEMINATION}

Written informed consent will be obtained from parents / guardians before the children join the cohort. Transportation expenses for participants to travel to GWCMC for neurological and cognitive assessments will be reimbursed. The protocol of this study has been approved by the ethics committee of GWCMC. Data usage follows the rules of hospital's data oversight committee. Biological samples will be handled after use following the national guideline on biological waste management and disposal. Findings of the GPCS-HFMD study will be disseminated through publications in international peer-reviewed journals and will be presented in academic conferences.

Acknowledgements The authors thank the participants of this study for their support.

Contributors $\mathrm{KL}$ and $\mathrm{XL}$ drafted the manuscript. $\mathrm{KL}$ contributed to the development of the statistical plan and statistical analysis of the retrospective data. XL contributed to the design of the cognitive assessments. YX, HL and HX contributed to the conception of the study and the development of a follow-up system. HL contributed to the training of research nurses. WS contributed to data management and prepared the retrospective data for analysis. All authors reviewed, read and approved the final manuscript.

Funding This work is supported by the Guangzhou Technology and Informatization Bureau (2014Y2-00197)

Competing interests None declared.

Patient consent for publication Not required.

Ethics approval Institutional Ethics Committee of the Guangzhou Women and Children's Medical Centre (2017102502).

Provenance and peer review Not commissioned; externally peer reviewed.

Open access This is an open access article distributed in accordance with the Creative Commons Attribution Non Commercial (CC BY-NC 4.0) license, which permits others to distribute, remix, adapt, build upon this work non-commercially, and license their derivative works on different terms, provided the original work is properly cited, appropriate credit is given, any changes made indicated, and the use is non-commercial. See: http://creativecommons.org/licenses/by-nc/4.0/.

\section{REFERENCES}

1. Zeng H, Lu J, Yang F, et al. The increasing epidemic of hand, foot, and mouth disease caused by coxsackievirus-A6, Guangdong, China, 2017. J Infect 2018;76:220-3.

2. Anh NT, Nhu LNT, Van HMT, et al. Emerging coxsackievirus a6 causing hand, foot and mouth disease, Vietnam. Emerg Infect Dis 2018;24:654-62.

3. Mirand A, Henquell C, Archimbaud C, et al. Outbreak of hand, foot and mouth disease/herpangina associated with coxsackievirus A6 and A10 infections in 2010, France: a large citywide, prospective observational study. Clin Microbiol Infect 2012;18:E110-8.

4. Fujimoto T, lizuka S, Enomoto M, et al. Hand, foot, and mouth disease caused by coxsackievirus A6, Japan, 2011. Emerg Infect Dis 2012;18:337-9.

5. Sinclair C, Gaunt E, Simmonds P, et al. Atypical hand, foot, and mouth disease associated with coxsackievirus A6 infection, Edinburgh, United Kingdom, January to February 2014. Euro Surveill 2014;19:20745.

6. Montes M, Artieda J, Piñeiro LD, et al. Hand, foot, and mouth disease outbreak and coxsackievirus A6, northern Spain, 2011. Emerg Infect Dis 2013;19.

7. Ang LW, Tay J, Phoon MC, et al. Seroepidemiology of coxsackievirus A6, coxsackievirus A16, and enterovirus 71 infections among children and adolescents in Singapore, 2008-2010. PLoS One 2015;10:e0127999.

8. Huang J, Liao Q, Ooi $\mathrm{MH}$, et al. Epidemiology of recurrent hand, foot and mouth disease, China, 2008-2015. Emerg Infect Dis 2018;24.

9. Deng T, Huang Y, Yu S, et al. Spatial-temporal clusters and risk factors of hand, foot, and mouth disease at the district level in Guangdong Province, China. PLoS One 2013;8:e56943.

10. Du Z, Xu L, Zhang W, et al. Predicting the hand, foot, and mouth disease incidence using search engine query data and climate variables: an ecological study in Guangdong, China. BMJ Open 2017;7:e016263.

11. Xing W, Liao Q, Viboud C, et al. Hand, foot, and mouth disease in China, 2008-12: an epidemiological study. Lancet Infect Dis 2014;14:308-18.

12. World health organization. A guide to clinical management and public health response for hand, foot, and mouth disease (hfmd): World health organization, 2011.

13. Kim B, Moon S, Bae GR, et al. Factors associated with severe neurologic complications in patients with either hand-foot-mouth disease or herpangina: a nationwide observational study in South Korea, 2009-2014. PLoS One 2018;13:e0201726. 
14. Sun BJ, Chen HJ, Chen $\mathrm{Y}$, et al. The risk factors of acquiring severe hand, foot, and mouth disease: a meta-analysis. Can J Infect Dis Med Microbiol 2018;2018:1-12.

15. Liu G, Xu Y, Wang X, et al. Developing a machine learning system for identification of severe hand, foot, and mouth disease from electronic medical record data. Sci Rep 2017;7:16341.

16. Mei L, Song X, Kong Y, et al. An assessment of a pediatric early warning system score in severe hand-foot-and-mouth disease children: To detect clinical deterioration in hospitalized children. Medicine 2018;97:e11355.

17. Zhang B, Wan X, Ouyang FS, et al. Machine learning algorithms for risk prediction of severe hand-foot-mouth disease in children. $\mathrm{Sci}$ Rep 2017;7:5368.

18. Chang LY, Huang LM, Gau SS, et al. Neurodevelopment and cognition in children after enterovirus 71 infection. N Engl J Med 2007;356:1226-34.

19. Huang MC, Wang SM, Hsu YW, et al. Long-term cognitive and motor deficits after enterovirus 71 brainstem encephalitis in children. Pediatrics 2006;118:e1785-8.

20. Guideline on hand-foot-and-mouth disease. Guideline on hand-footand-mouth disease diagnosis and treatment 2018. 2018. (in chinese)

21. Gesell inst. Of human development. New Haven, CT, USA: Gesell inst.

22. W D. Wechsler intelligence scale for children. 4th edn. san antonia, tx: Psychcorp, 2003

23. Harrison POT. Adaptive behavior assessment system. 3rd edn, 2015.

24. Frankenburg WK, Dodds J, Archer P, et al. The denver ii: a major revision and restandardization of the denver developmental screening test. Pediatrics 1992;89:91-7.
25. Li MZ, Pang LL, Bai AY, et al. Association of chemotactic chemokine ligand 5 polymorphisms with the risk of developing severe enterovirus 71 infection. Am J Trop Med Hyg 2015;93:709-13.

26. Chang LY, Chang IS, Chen WJ, et al. HLA-A33 is associated with susceptibility to enterovirus 71 infection. Pediatrics 2008;122:1271-6.

27. Zhao N, Chen HL, Chen ZZ, et al. IL-10-592 polymorphism is associated with IL-10 expression and severity of enterovirus 71 infection in chinese children. J Clin Virol 2017;95:42-6.

28. Jones E, Pillay TD, Liu F, et al. Outcomes following severe hand foot and mouth disease: a systematic review and meta-analysis. Eur $J$ Paediatr Neurol 2018;22:763-73.

29. Chou IC, Lin CC, Kao CH. Enterovirus encephalitis increases the risk of attention deficit hyperactivity disorder: a taiwanese populationbased case-control study. Medicine 2015;94:e707

30. Gau SS, Chang LY, Huang LM, et al. Attention-deficit/hyperactivityrelated symptoms among children with enterovirus 71 infection of the central nervous system. Pediatrics 2008;122:e452-8.

31. Akcakaya NH, Tekturk P, Cagatay A, et al. Atypical enterovirus encephalitis causing behavioral changes and autism-like clinical manifestations: case report. Acta Neurol Belg 2016;116:679-81.

32. Marques F, Brito MJ, Conde M, et al. Autism spectrum disorder secondary to enterovirus encephalitis. J Child Neuro 2014;29:708-14.

33. Goto K, Sanefuji M, Kusuhara K, et al. Rhombencephalitis and coxsackievirus A16. Emerg Infect Dis 2009;15:1689-91.

34. Lo SH, Huang YC, Huang CG, et al. Clinical and epidemiologic features of coxsackievirus A6 infection in children in northern Taiwan between 2004 and 2009. J Microbiol Immunol Infect 2011:44:252-7. 